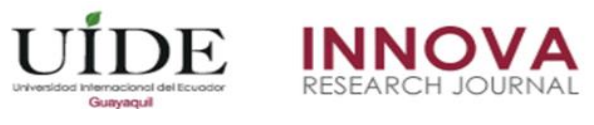

INNOVA Research Journal, ISSN 2477-9024

(Enero, 2017). Vol. 2, No.1 pp. 34-44

DOI: https://doi.org/10.33890/innova.v2.n1.2017.111

URL: http://revistas.uide.edu.ec/index.php/innova/index

Correo: innova@uide.edu.ec

\title{
Propuesta de productos alimenticios no tradicionales de frejol
}

\section{Proposal of non-traditional bean food products}

Nelly Sayenka Vergara Díaz

Julio Terranova Mera

Universidad de Guayaquil, Ecuador

Fernando Rodolfo Orellana Intriago

Víctor Hugo Vizueta Tomalá

Daniel Antonio Mata López

David Alberto Bernal Paredes

Pablo Ricardo San Andrés Reyes

Universidad Ecotec, Ecuador

Autor para correspondencia: nellysvergarad@gmail.com, julio_terranova@hotmail.com, fernando.orellanai@ug.edu.ec,vizuetavh@hotmail.com,daniel.matal@ug.edu.ec,

davidbernalparedes@gmail.com, psanandres@ecotec.edu.ec.

Fecha de recepción: 06 de Noviembre de 2016 - Fecha de aceptación: 20 de Diciembre de 2016.

Resumen: En Ecuador existen cultivos de diversas clases de fréjol negro, pero los principales son la calima y la caraota, esta última es conocida en el país como fréjol negro, siendo muy apetecida en países como México, Brasil, Colombia y Venezuela. Ecuador produce principalmente este producto para la exportación, siendo la exportación de este producto realizada al mercado europeo. La demanda en el mercado europeo se ha incrementado, principalmente por el valor que los consumidores de este mercado brindan a su valor nutritivo, ya que este producto contiene carbohidratos, proteínas, vitaminas A y B, calcio, magnesio, fósforo, potasio, entre otros. Actualmente, dado el mayor volumen y difusión de estudios científicos, por ejemplo el de la Organización Mundial de la Salud sobre el potencial peligro para la salud debido al consumo de embutidos y carne roja, el consumidor está demandando mayor variedad de productos que puedan ser nutritivos y a la vez deliciosos, por lo cual, siendo el fréjol negro rico en proteínas y nutrientes se convierte en una alternativa ideal, cuyo mercado se expande incluso a aquellos consumidores con necesidades especiales, y que demandan productos sin gluten ni lactosa, por lo que la apuesta a ofrecer productos derivados del fréjol negro, como harinas, galletas y derivados saludables (sin azúcar, integrales, bajas en sal o sin grasas trans) tienen una oportunidad de crecimiento a nivel interno, pero principalmente a la exportación.

Palabras Clave: Cultivos; Productos Alimenticios No Tradicionales; Fréjol Negro

Abstract: In Ecuador there are crops of different kinds of black beans, but the main ones are the Calima and caraota, the last one is known in the country as black beans, being very sought in countries like Mexico, Brazil, Colombia and Venezuela. They are produced mainly to export to European market, where demand has increased, principally because of the value that consumers provide to its nutritional value, as this product contains carbohydrates, protein, vitamins A and B, calcium, magnesium, phosphorus, potassium, among others. Currently, given the greater volume

and dissemination of scientific studies, such as the World Health Organization about the potential 
danger to health due to the consumption of sausages and red meat. Consumers are demanding greater variety of products that can be nutritious and delicious, so, being protein-rich food becomes an ideal alternative, whose market expands even those clients with special needs, and demand products without gluten and lactose, that's why commitment to offer products derived from black beans, such as flour, biscuits and derivatives healthy (without sugar, whole, low salt or no transfat) have an opportunity to grow internally, but especially for export.

Key Words: Crops; Non-Traditional Food Products; Bean

\section{Introducción}

En Ecuador se cultivan varias clases de fréjol negro. Las más populares son el calima y la caraota. Esta especie es muy apetecida en países como Cuba, México, Brasil, Colombia y Venezuela. En el país, también se lo consume aunque la producción está direccionada básicamente para la exportación a Europa, donde tiene creciente demanda. Se cultiva en climas cálido y templado.

Su valor nutritivo El fréjol posee carbohidratos, proteínas, vitaminas A y B, calcio, magnesio, fósforo, potasio, entre otros. En el Ecuador, principalmente en la región Sierra, las leguminosas son componentes de los sistemas de producción, ya que son cultivadas en asociación, intercaladas, en monocultivos o en rotación con otros cultivos; por tal motivo juega un papel muy importante en el manejo sostenible de la agricultura y la alimentación, por lo que genera empleo, alimento e ingresos económicos a pequeños, medianos y grandes agricultores, que tratan de satisfacer la demanda interna y externa.

Desde el año 2000 al 2012, la producción nacional de fréjol seco registró una disminución de $44.65 \%$. En el censo del año 2000 se reportó una producción de 18 mil toneladas y en la encuesta del 2012 (ESPAC-INEC) se redujo a 9.99 mil toneladas.

En el censo del año 2000 se registró una superficie cosechada de frejol seco de alrededor de 89.7 mil hectáreas, mientras que en la encuesta del 2012 (ESPAC-INEC) se reportó una superficie de 35 mil hectáreas, lo que significa una reducción de $61 \%$. Cabe destacar, que en este mismo período la productividad aumento en un $42 \%$ (de 0.20 a $0.28 \mathrm{tm} / \mathrm{ha}$ ). Si bien la productividad de fréjol seco ha crecido $(0.28 \mathrm{tm} / \mathrm{a})$ en el último decenio, dista mucho de los niveles de productividad alcanzado por Colombia y Perú; quienes alcanzan promedios de 1.03 y $1.13 \mathrm{tm} / \mathrm{ha}$, respectivamente, con una extensión de 125 mil hectáreas y una producción de 128 mil toneladas en Colombia y una extensión de 82 mil hectáreas y 92 mil toneladas producidas en Perú.

Para el 2013, aun sin datos oficiales, se estima en 9.7 mil toneladas la de fréjol seco; un $41 \%$ por debajo del máximo alcanzado en el 2005 (23.5 mil toneladas de fréjol seco). La caída se explica mayormente por la disminución del área cosechada a partir del año 2005. Por otra parte, la provincia de Chimborazo es la provincia con mayor producción para el fréjol seco (19\%). Cuenta con el mayor nivel de productividad del país $(0.49 \mathrm{tm} / \mathrm{ha})$ con una superficie cosechada 3.9 mil hectáreas. Por otro lado, Azuay es la provincia de más baja productividad $(0.14 \mathrm{tm} / \mathrm{ha})$, produce cerca del $15 \%$ de la producción nacional y cuenta con la mayor superficie cosechada, 11.2 mil hectáreas. 


\section{Métodos}

Para esta investigación se utilizará el método deductivo que nos permitirá interpretar y comprender las particularidades del tema planteado. Se utilizarán la investigación documental y descriptiva considerando que tenemos como objeto de estudio la producción de soya en el Ecuador y sus oportunidades en el mercado mundial. La Investigación descriptiva "Se reseñan la características o rasgos de la situación o fenómeno objeto del estudio" (Bernal, Cesar A, 2010) y la Investigación Documental "La investigación documental consiste en un análisis de la información escrita sobre un determinado tema, con el propósito de establecer relaciones, diferencias, etapas, posturas o estado actual del conocimiento respecto al tema objeto del estudio.” (Bernal, Cesar A, 2010). A través de la obtención de la información para la investigación por medio de la observación, internet y análisis de documental del tema planteado y a través de los datos obtenidos de la página de páginas web.

\section{Resultados}

\section{Tendencias de la Demanda}

\section{Exportaciones}

El comercio internacional del Ecuador en el rubro fréjol, presenta una tendencia al alza en las exportaciones, registrado un incremento de $61 \%$ en el periodo 2000 - 2013. En el inicio de este período, Ecuador exportó 7.75 mil toneladas, registrándose el pico máximo en el año 2005 con 27.41 mil toneladas y a partir de ese año, las exportaciones se redujeron considerablemente hasta el año 2013, donde se reportó una cantidad de 12.5 mil toneladas. Esta reducción se debió principalmente a la disminución en la producción de fréjol a nivel nacional.

En relación a las importaciones, estas han sido históricamente menores a las exportaciones, registrándose así una balanza comercial positiva. En el año 2000, el país importó 97 toneladas y en el 2013, 810 toneladas. En el 2013 las exportaciones se concentraron en el último cuatrimestre del año (septiembre-diciembre) en donde se exportó el $57 \%$ de este producto, cuyos principales destinos fueron Colombia y Estados Unidos.

\section{Precios}

El precio al productor en el año 2013 del fréjol seco variedad "Gema" osciló entre39.37 (noviembre) y 67.27 (junio) USD/qq, con un precio promedio de 54.69 USD/qq, un $16 \%$ mayor al precio promedio del 2012 (47.33 USD/qq). A nivel de mercado mayorista, el precio promedio registrado en las ciudades de Guayaquil y Cuenca fue de 141.36 USD/qq. El precio más alto pagado a nivel mayorista fue de 199 dólares por bulto de fréjol, registrado en el mes de marzo. 


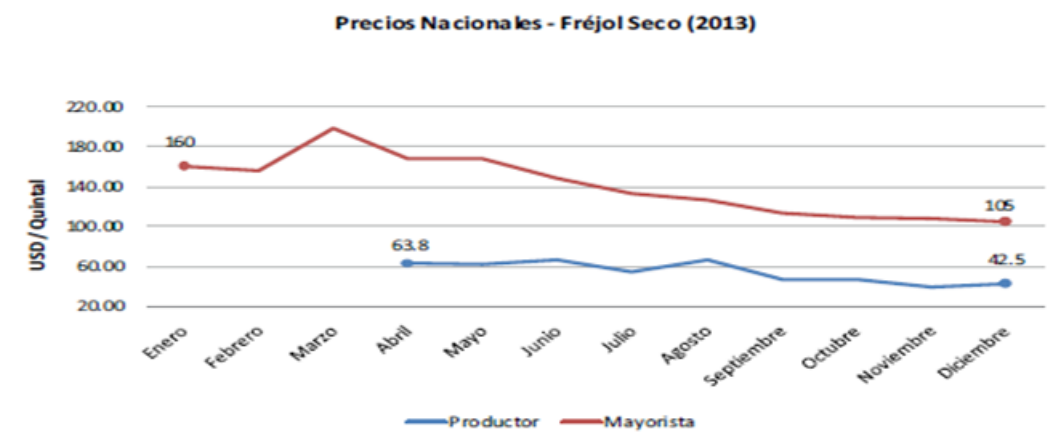

Fuente: SINAGAP

\section{Importaciones}

El $8.1 \%$ de la producción mundial de fréjol seco es comercializada (1.91 MM), lo cual indica que la mayoría de la producción es para el consumo interno. Los mayores importadores de este grano son Brasil, México e Italia, en su conjunto captan el 34\% del comercio mundial; gran parte de la demanda es abastecida por China.

\section{Estructura de las Importaciones Mundiales}

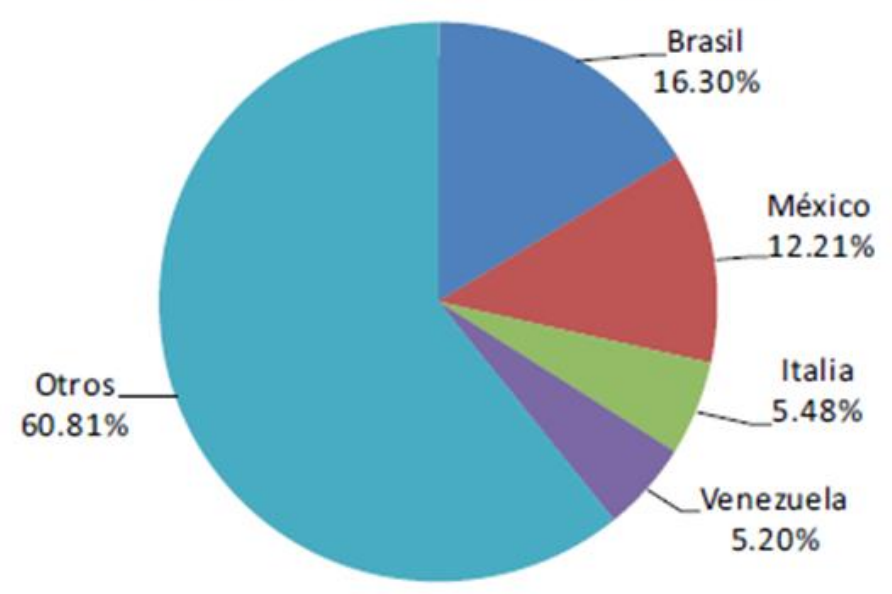

Fuente: TRADE MAP, 2012

Las importaciones en el año 2013 fueron menores a las exportaciones (810 toneladas), la mayor cantidad de estas se registró entre los meses de mayo a agosto (83\% de las exportaciones totales). El 90\% del total de las importaciones tuvieron como origen Perú. 


\section{Importaciones y Exportaciones Toneladas (2013)}

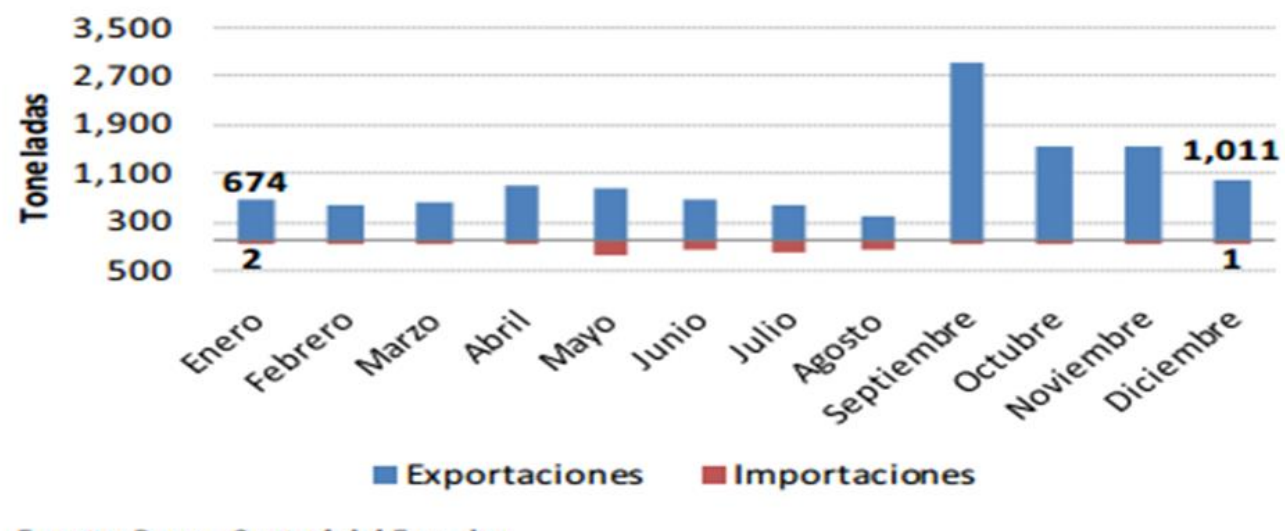

Fuente: Banco Central del Ecuador

\section{Datos Socioeconómicos}

\section{Población Italiana}

Italia, situada en el sur de Europa, tiene una superficie de $301.340 \mathrm{Km} 2$.

Italia, con una población de 60.594 .854 personas, se encuentra en la $23^{\circ}$ posición de la tabla de población, compuesta por 196 países y una densidad de población de 202 habitantes por $\mathrm{Km} 2$.

Su capital es Roma y su moneda Euros.

Italia es la $8^{\text {a }}$ economía por volumen de PIB. Su deuda pública en 2014 fue de 2.135 .902 millones de euros, un 132,30\% del PIB y su deuda per cápita de $35.060 €$ euros por habitante.

\begin{tabular}{ll}
\hline $\mathbf{6 0 5 9 4 8 5 4}$ & Población actual \\
\hline $\mathbf{2 9} 669487$ & Población masculina actual $(49.0 \%)$ \\
\hline $\mathbf{3 0 9 2 5 3 6 7}$ & Población femenina actual (51.0\%) \\
\hline $\mathbf{1 0 6} 116$ & Nacimientos este año \\
\hline $\mathbf{1 2 9 4}$ & Nacimientos hoy \\
\hline $\mathbf{1 1 3 7 4 6}$ & Muertes este año \\
\hline $\mathbf{1 3 8 7}$ & Muertes hoy \\
\hline $\mathbf{5 6 ~ 1 7 9}$ & La migración neta este año \\
\hline $\mathbf{6 8 5}$ & La migración neta hoy \\
\hline $\mathbf{4 8 5 5 0}$ & Crecimiento poblacional este año \\
\hline $\mathbf{5 9 2}$ & Crecimiento poblacional hoy \\
\hline
\end{tabular}

\section{Datos Demográficos}




\section{Italia}
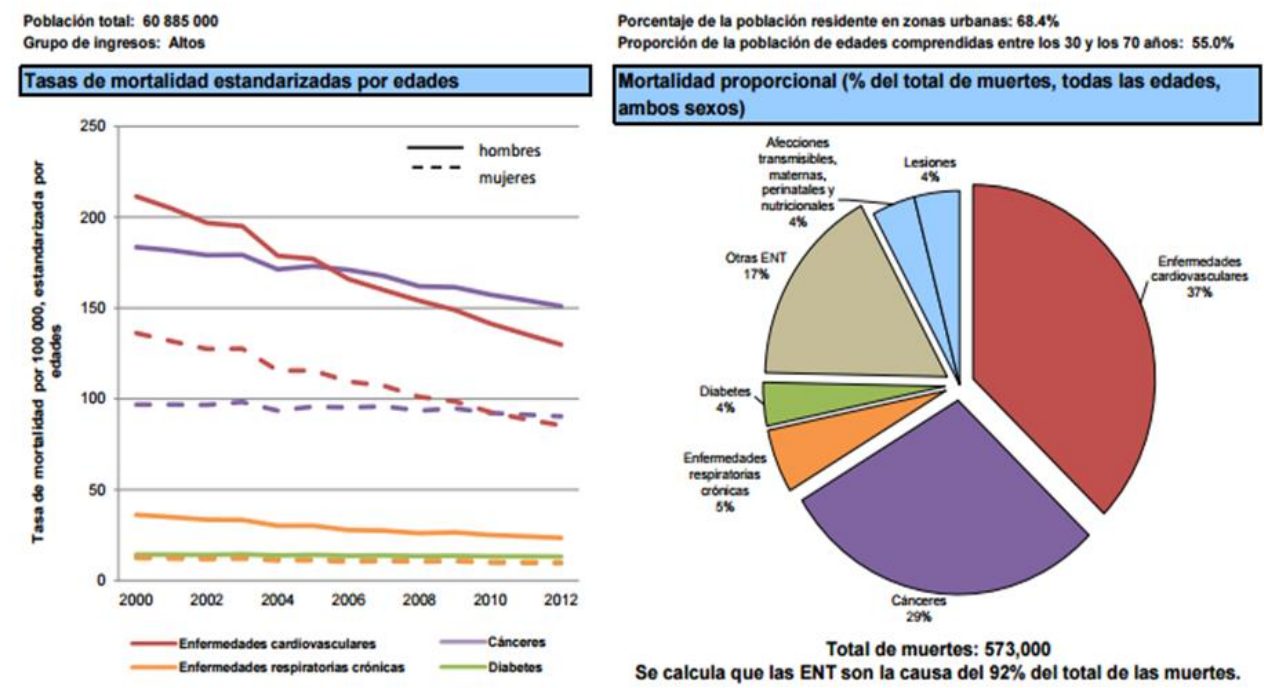

Las tasas de mortalidad normalizadas por cáncer, cardiopatías isquémicas y accidentes de transporte siguieron una tendencia a la baja entre 2004 y 2012.

Entre 2004 y 2012 se produjo una reducción del 10,2\% en las tasas de mortalidad normalizadas relacionadas con el cáncer en el caso de los hombres y del 5,5\% en el caso de las mujeres. Se registraron reducciones mayores en relación con las muertes por cardiopatías isquémicas, cuyas tasas de mortalidad experimentaron un descenso del $28,5 \%$ para los hombres y del $30,4 \%$ para las mujeres, mientras que se registraron reducciones aún mayores en las muertes por accidentes de transporte, cuyas tasas se redujeron en un 40,8\% para los hombres y un 43,8\% para las mujeres. La tasa de mortalidad normalizada por cáncer de mama se redujo en un 9,8\% para las mujeres, valor superior al cambio general experimentado en el caso del cáncer.

Por contra, las tasas de mortalidad por enfermedades del sistema nervioso aumentaron para los hombres en un $16,3 \%$ y para las mujeres en un 23,3\%. Existe una serie temporal más corta disponible para el cáncer de pulmón (incluido también el cáncer de tráquea y bronquios) que muestra tendencias divergentes: en el caso de los hombres, la tasa de mortalidad normalizada disminuyó un 4,9\%, mientras que entre las mujeres aumentó un 5,7\%.

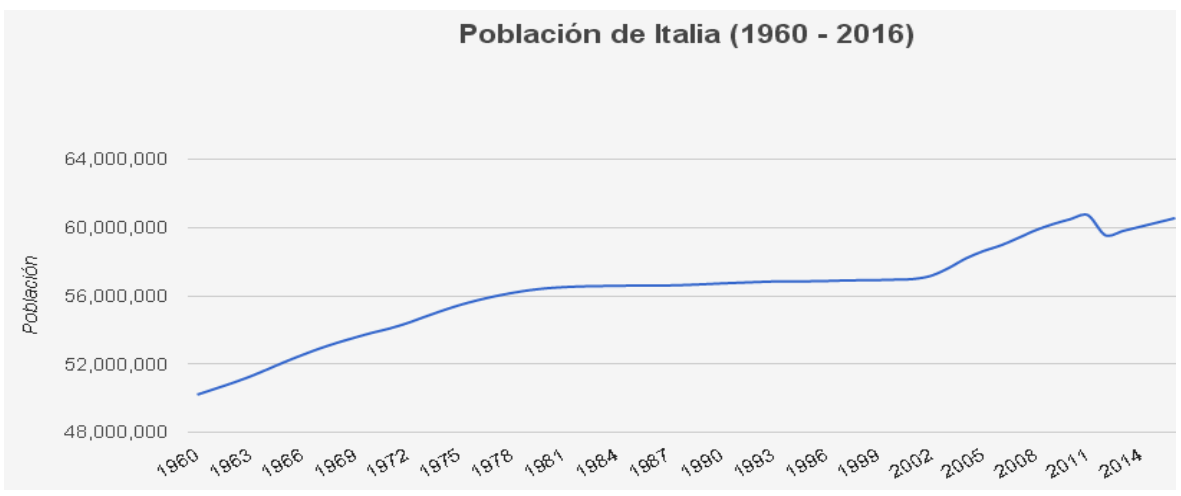




\section{Características De Los Consumidores}

Se ha elegido el mercado Italiano ya que según los datos de Caobisco, un grupo de países como Italia, Francia y Holanda tienen un consumo entorno a los 10 kilos per cápita en lo que respecta a galletas.

En lo que respecta al frejol el cuadro $\mathrm{N}^{\mathrm{0}} 11$ detalla los acuerdos comerciales suscritos por Ecuador, aplicables a la partida 071333 , correspondiente a fréjol.

Cuadro No. 11

\begin{tabular}{|l|l|}
\hline \multicolumn{2}{|c|}{ ACUERDOS COMERCIALES PARA LA PARTIDA: 0713.33 } \\
\hline \multicolumn{1}{|c|}{ PAIS } & ACUERDO \\
\hline Italia & MFN duties (Applied) \\
\hline México & MFN duties (Applied) \\
\hline Reino Unido & Preferential tariff for CAN countries \\
\hline Venezuela & MFN duties (Applied) \\
\hline España & Preferential tariff (AAP.CE59) for Ecuador \\
\hline Brasil & MFN duties (Applied) \\
\hline Sudafrica & Preferential tariff(AAP.AG2) for Ecuador /Preferential tariff \\
\hline Cuba & (AAP.CE46) for Ecuador \\
\hline Francia & MFN duties (Applied) \\
\hline Argelia & General tariff \\
\hline India & MFN duties (Applied) \\
\hline Turquía & MFN duties (Applied) \\
\hline Países Bajos (Holanda) & MFN duties (Applied) \\
\hline Estados Unidos de América & Andean Trade Preference Act / Preferential tariff for GSP countries \\
\hline Portugal & MFN duties (Applied) \\
\hline Pakistan & MFN duties (Applied) \\
\hline Bélgica & MFN duties (Applied) \\
\hline Emiratos Árabes Unidos & MFN duties (Applied) \\
\hline Colombia & Preferential tariff for CAN countries \\
\hline Grecia & MFN duties (Applied) \\
\hline República Dominicana & MFN duties (Not Applied) \\
\hline Perú & Preferential tariff for CAN countries \\
\hline & Parket Access Map \\
\hline & Fón: CICO-CORPEI \\
\hline
\end{tabular}

\section{Barreras Arancelarias}

En el cuadro se detallan los aranceles promedio que los principales países importadores de fréjol aplican al producto proveniente de Ecuador para su ingreso. La tarifa aplicada corresponde a la que el producto efectivamente debe pagar al ingresar al país y la tarifa advalorem es un promedio calculado en base a tarifas específicas, mixtas, antidumping y cuotas. 


\begin{tabular}{|c|c|c|}
\hline \multicolumn{3}{|c|}{ ARANCEL PROMEDIO PARA LA PARTIDA: 0713.33} \\
\hline Principales importadores mundiales & Aplicada & Ad-valorem \\
\hline Italia & $0.00 \%$ & $0.00 \%$ \\
\hline México & $93.83 \%$ & $125.10 \%$ \\
\hline Reino Unido & $0.00 \%$ & $0.00 \%$ \\
\hline Venezuela & $0.00 \%$ & $0.00 \%$ \\
\hline España & $0.00 \%$ & $0.00 \%$ \\
\hline Brasil & $0.00 \%$ & $0.00 \%$ \\
\hline Sudafrica & $10.00 \%$ & $10.00 \%$ \\
\hline Cuba & $0.00 \%$ & $0.00 \%$ \\
\hline Francia & $0.00 \%$ & $0.00 \%$ \\
\hline Argelia & $5.00 \%$ & $5.00 \%$ \\
\hline India & $10.00 \%$ & $10.00 \%$ \\
\hline Turquía & $9.65 \%$ & $19.30 \%$ \\
\hline Paises Bajos (Holanda) & $0.00 \%$ & $0.00 \%$ \\
\hline Estados Unidos de América & $0.00 \%$ & $0.00 \%$ \\
\hline Portugal & $0.00 \%$ & $0.00 \%$ \\
\hline Pakistan & $0.00 \%$ & $0.00 \%$ \\
\hline Bélgica & $0.00 \%$ & $0.00 \%$ \\
\hline Emiratos Arabes Unidos & $2.50 \%$ & $5.00 \%$ \\
\hline Colombia & $0.00 \%$ & $0.00 \%$ \\
\hline Grecia & $0.00 \%$ & $0.00 \%$ \\
\hline Republica Dominicana & $25.00 \%$ & $25.00 \%$ \\
\hline Perú & $0.00 \%$ & $0.00 \%$ \\
\hline
\end{tabular}

\section{Competencia}

Existen algunas empresas dedicadas a la elaboración de galletas en el mercado italiano, pero no de frejol, al analizar el segmento de mercado de galletas, los datos obtenidos del Panel de Consumo Alimentario del Ministerio de Agricultura, Alimentación y Medio Ambiente, sitúan las ventas totales de galletas en 2012 en 236.914 toneladas y 834,5 millones de euros, frente a las 229.244 t y 762,1 millones de euros de 2011. Las galletas envasadas totalizaron 232.624 t y 794 millones de euros, con un precio medio de 3,41 euros por $\mathrm{kg}$, con un consumo por persona y año de $5,05 \mathrm{~kg}$, y un gasto medio de 17,2 euros. De ese total, las dulces sumaron $224.994 \mathrm{t}(+2,3 \%) \mathrm{y}$ 760,5 millones de euros $(+5,9 \%)$, las saladas 7.630 t y 33,8 millones de euros, y las dietéticas 4.129 t y 24 millones de euros. Las ventas de galletas a granel realizadas en establecimientos de impulso y pastelería totalizaron 4.290 t y 40,2 millones de euros. El precio medio de esta modalidad fue de 9,38 euros/kg.

\section{Normas Legales y Políticas}

El tener una estabilidad política es de vital importancia para que la inversión extranjera y privada se sienta confiada en invertir en nuestro país, la implementación de políticas de empleo para alcanzar un desarrollo sostenible que beneficien a empleados sin entorpecer a las industrias permite lograr un crecimiento económico en un ambiente legal y justo.

El Ecuador, al igual que la mayor parte de los países latinoamericanos, ha logrado establecer un marco institucional moderno y especializado, basado en la promulgación de la ley de comercio exterior e inversiones.

A través de incentivos a la producción como las nuevas reformas tributarias vigente que contiene básicamente incentivos fiscales:

- Exoneración del Impuesto a la Renta. 
- Deducción del Impuesto a la Renta.

- Exoneración de impuestos para las actividades de energía renovable.

- Exoneración del Impuesto a la Renta a la inversión productiva en los sectores priorizados.

- Deducción del gasto en nueva mano de obra en nuevos emprendimientos en zonas de menor desarrollo por los primeros años.

- Zonas especiales de desarrollo económico (ZEDE)

\section{Demanda}

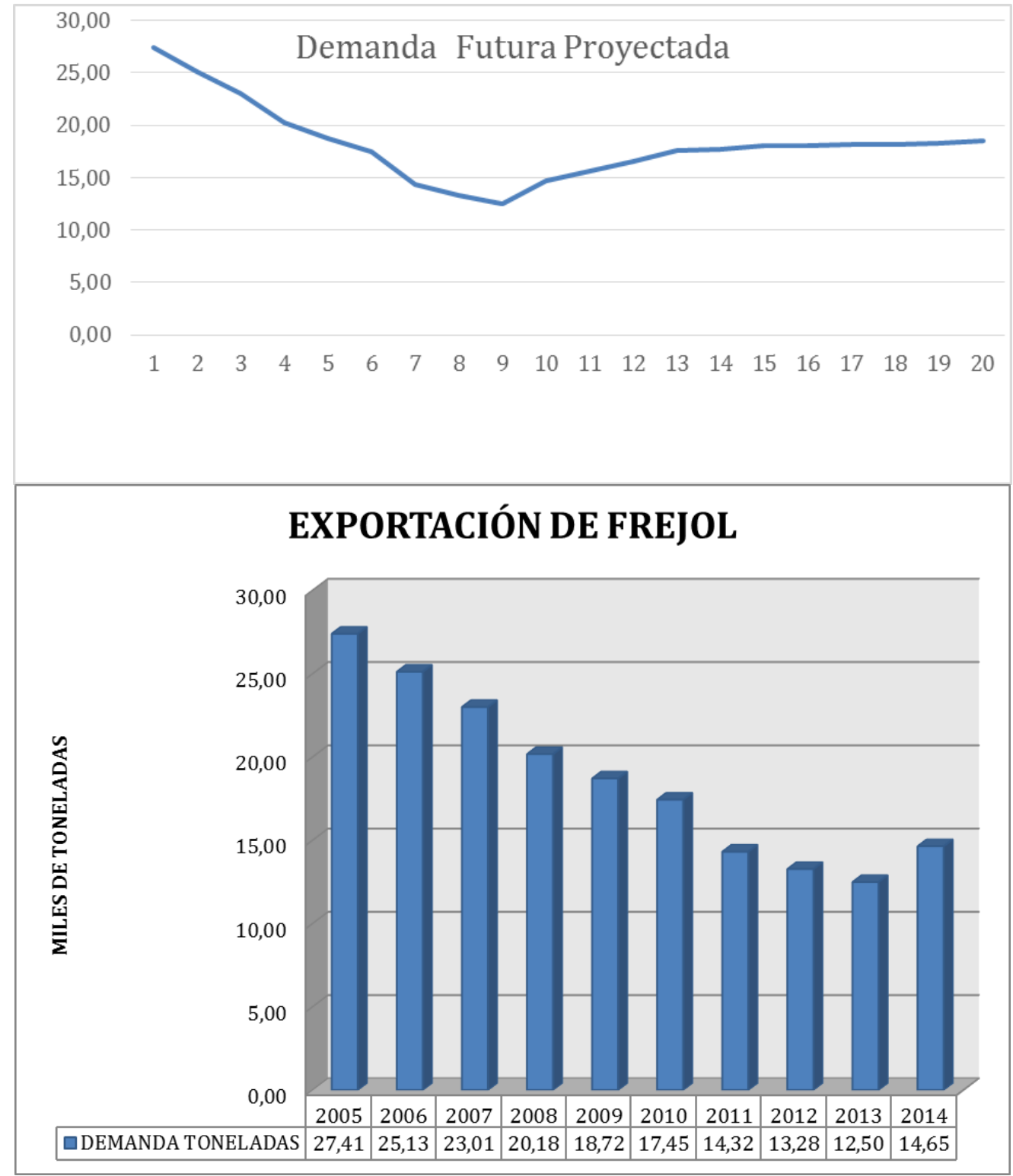




\section{Conclusiones}

Para poder satisfacer las nuevas y cambiantes demandas de los consumidores, y dándole además un mayor valor agregado debido a las proteínas y nutrientes que contiene el frejol negro; además de prestar especial atención a los clientes con necesidades especiales y que demandan productos sin gluten y sin lactosa, se apuesta el ofrecer galletas más saludables (sin azúcar, integrales, bajas en sal o sin grasas trans) como son Las Galletas De Frejol Negro.

Por eso se ha considerado el frejol negro ya que pueden proveernos de todos los aminoácidos que el organismo necesita, contienen más antioxidantes que ninguna otra legumbre, eliminan los radicales libres y previenen el crecimiento de células cancerígenas en el cuerpo, así como la formación de distintas enfermedades, son ricos en fibra, lo que los hace ideales para los pacientes diabéticos y con metabolismos de glucosa irregulares, regula el aparato digestivo y previene los constipados, es ideal para las personas que padecen divertículos y otros trastornos en el colon favorecen su curación incluyendo dicha legumbre en su dieta habitual.

\section{Bibliografía}

Agrodigital. (2015). Obtenido de http://www.agrodigital.com/

BCE, B. C. (2015). Reporte de Coyuntura.

Bernal, Cesar A. (2010). Metodología de la Investigación. En C. A. Bernal, Metodología de la investigación (pág. 111). Bogotá: Orlando Fernández.

Bernal, Cesar A. (2010). Metodología de la Investigación. En B. Cesar, Metodología de la Investigación. Bogotá: Orlando Fernández.

Cultivos, P. p. (2015). FAO. Obtenido de http://www.fao.org/docrep/004/y3557s/y3557s08.htm Ecuatoriano, C. N. (2014). Informe.

Encuesta de Superficie, y. P. (2014). Instituto Nacional de Estadística y Censos. FAO. (2015). Perspectivas por sectores principales. Producción de cultivos. Obtenido de http://www.fao.org/worldfoodsituation/csdb/es/

Foodways, F. (2015). Consumo Humano.

Malthus, T. (1766-1834).

Ministerio de Agricultura, G. A. (2015). Informe de Rendimientos Y Características De Soya En El Ecuador Verano. Guayaquil.

ONU, O. d. (2015). Informe de perspectivas de la población mundial.

Soja Producción Mundial. (Septiembre 2016 de 2016). Https: 
//www.produccionmundialsoja.com/, online. (S. P. Mundial, Editor, P. J. 2016, Productor, \& Soja Producción Mundial) Recuperado el 3 de agosto de 2016, de https://www.produccionmundialsoja.com/: https://www.produccionmundialsoja.com/

Unión Vegetariana Internacional. (2016). Obtenido de http://www.ivu.org/congress/wvc08/: http://www.ivu.org/congress/wvc08/ 